\title{
ISSN 0004-9727
}

\section{BULLETIN Volume 11 (1974)}

of the Australian Mathematical Society

Number 2

K. John and V. Zizler Duals of Banach spaces which admit nontrivial smooth functions

Kusum Soni and

R. P. Soni

Edward D. Fackerell and R. A. Littler

P. B. Kirkpatrick

A. Shafaat

T. R. Gulati

A. M. Russell

David A. Birnbaum James R. Boone K. K. Puri

D. W. Barclay and

T. Bryant Moodie

A tauberian theorem related to the modified Hankel transform

Polynomials biorthogonal to Appell's polynomials

Collineation groups which are sharply transitive on an oval

On varieties closed under the construction of power algebras

A Fritz John type sufficient optimality theorem in complex space

An integral representation for a generalised variation of a function

Preregular maps between Banach lattices

Concerning collectionwise Hausdorff spaces

Waves on a shear flow

Verifications of a formal technique for viscoelastodynamics

Abstracts of Australasian PhD theses

Michael Donald

Hendy

M. A. Lohe

Walter R. Bloom

J. L. Davy

Susan Dancs Groves
Finite evaluation of the class number of quadratic number fields

The development of the boson calculus for the orthogonal and symplectic groups

Bernstein's inequality for locally compact groups

Generalized differential equations satisfying

Carathéodory type conditions

Locally finite near-fields
309

311

315

317

319

Bull. Austral. Math. Soc. Vol. 11 (1974) No. 2, pp. 161-320

\section{THE UNIVERSITY OF QUEENSLAND PRESS \\ ST LUCIA, QUEENSLAND 4067}




\title{
THE AUSTRALIAN MATHEMATICAL SOCIETY
}

\author{
President: H. S. Green University of Adelaide \\ Secretary: W. Pye Melbourne State College, \\ 757 Swanston Street, \\ Carlton, Victoria 3053, Australia \\ Treasurer: V. G. Hart Department of Mathematics, \\ University of Queensland \\ St Lucia, Queensland 4067, Australia. \\ (These officers serve until May 1975)

\section{Membership and correspondence}

Applications for membership, notices of change of address or title or position, and other correspondence, except as noted below, should be sent to the Secretary (address above). Subscriptions and correspondence related to accounts should be sent to the Treasurer (address above). Correspondence about the distribution of the Society's JOURNAL, BULLETIN, and GAZETTE and orders for back numbers, should be sent to the Business Manager, whose address is the same as the Treasurer's (above).

\section{Publications}

The JOURNAL of the Australian Mathematical Society began publication in 1959 , and now appears in two volumes, each of four parts, annually. Manuscripts for publication should be sent to the Editor:

Professor J. N. Crossiey

Department of Mathematics,

Monash University,

Clayton, Victoria 3168, Australia,

or to one of the Associate Editors listed in a recent part of the JOURNAL. The BULLETIN of the Australian Mathematical Society began publication in 1969. Two volumes of three numbers each are published annually. Manuscripts for publication should be sent to the Editor:

Professor B. H. Neumann,

Department of Mathematics,

Institute of Advanced Studies,

Australian National University,

POB4, Canberra, ACT 2600, Australia,

or to the Associate Editor, Dr L. G. Kovács, at the same address.

The Australian Mathematical Society GAZETTE started publication in 1974, and carries news items, mathematical articles of general interest, and articles on tertiary mathematics teaching. Manuscripts for publication should be sent to the Editor at the

School of Mathematics,

University of New South Wales,

Kensington, NSW 2033, Australia. 